\title{
Synthesis and antimicrobial screening of some new pyrimido[1,2-a]benzimidazole derivatives
}

\author{
Nirav M. Shah*, Hitendra S. Joshi \\ Department of Chemistry, Saurashtra University, Rajkot - 360 005, Gujarat, India \\ *E-mail address: nirav.shah.m@gmail.com
}

\begin{abstract}
Some new pyrimido[1,2-a]benzimidazole derivatives were synthesized by reacting 2 -amino benzimidazole and chalcones in n-butanol at reflux temperature. In our present study we have used various heterocyclic chalcones derived from furfural and substituted acetophenones. All synthesized compounds were characterized by IR, ${ }^{1} \mathrm{H}$ NMR and Mass spectroscopy. All synthesized compounds were screened for their antimicrobial activity against gram positive and gram negative bacteria which showed moderate to good activity.
\end{abstract}

Keywords: 2-amino benzimidazole; pyrimido[1,2-a]benzimidazole; chalcone; antimicrobial activity azoloazine

\section{INTRODUCTION}

In recent years, organic research for synthesis of novel nitrogen containing heterocyclic ring system is emerging. Over the years of active research, benzimidazole pharmacophore in many major drugs like albendazole, mebendazole, thiabendazole as antihelmintics; omeprazole, lansoprazole, pantoprazole as proton pump inhibitors; astemizole as antihistaminic; enviradine as antiviral; candesarten cilexitil and telmisartan as antihypertensives and many lead compounds in a wide range of other therapeutic areas ${ }^{1-7}$.

Pyrimidine ring and its derivatives have been studied for therir chemical and biological singnificance from several decades. Pyrimidine derivatives have been reported as antibacterial, antiviral, and antitumor agents ${ }^{8}$.Various fused heterocyclic ring system with pyrimidine nucleus are known for their significant biological activities 9 .

Binucleophiles of aminoazole type are quite important reagents in modern heterocyclic chemistry, and their reactions with electrophiles are the most widespread and facile synthetic approach for obtaining diverse heterocyclic systems containing azole moiety. The most investigated area of aminoazole chemistry is their two-component reactions with ketoesters, $\beta$-dicarbonyls or $\alpha, \beta$-unsaturated aldehydes and ketones yielding fused azoloazines ${ }^{10-11}$.

Our research group is associated with synthesis and evaluation of biological activities of pyrimidine, pyridine and related nitrogen containing heterocyclic ring system from last decade $^{12-16}$. As from the above facts of medicinal importance of benzimidazole and pyrimidine ring system, we have planned to synthesized some new derivatives of 
pyrimido[1,2-a]benzimidazole and evaluate its antimicrobial activities as compare to standard drugs.

In present research work, we submitted simple, rapid and catalyst free synthesis of some new derivatives of 1,4-dihydro pyrimido[1,2-a]benzimidazole by reacting 2-amino benzimidazole and various $\alpha, \beta$-unsaturated ketone (chalcone) in n-butanol solvent at reflux temperature ${ }^{17-21}$.

\section{EXPERIMENTAL}

All chemicals and solvents were purchased from Spectrochem Pvt Ltd., Mumbai of AR grade and were used without further purification. Melting points were taken in open capillary method and are uncorrected. IR spectra were recorded on FTIR-8400 spectrophotometer (Shimadzu, Kyoto, Japan), using DRS prob KBr pallet. ${ }^{1} \mathrm{H}-\mathrm{NMR}$ spectra of the synthesized compounds were recorded on a Bruker-Avance-II $(400 \mathrm{MHz}) \mathrm{DMSO}_{6}$ solvent. Chemical shifts are expressed in $\delta \mathrm{ppm}$ downfield from TMS as an internal standard. Mass spectra were determined using direct inlet probe on a GCMS-QP 2010 mass spectrometer (Shimadzu, Kyoto, Japan).

\section{1. General procedure for synthesis of (2E)-1-(furan-2-yl)-3-substituted phenyl prop-2- en-1-one (1a-i)}

A solution of substituted acetophenones $(0.01 \mathrm{~mol})$ in ethanol $(10 \mathrm{ml})$ was added to a solution of furfural $(0.01 \mathrm{~mol})$ in ethanol $(10 \mathrm{ml})$. To this mixture $40 \% \mathrm{NaOH}$ solution was added drop wise as to make it just alkaline $(\mathrm{pH} 10 \sim 11)$. The reaction mass was stirred for 18 hrs at room temperature. The product was isolated by filtration and crystallized using appropriate solvent.

\section{2. General procedure for the synthesis of 4-(furan-2-yl)-2-substituted phenyl-1,4- dihydro pyrimido[1,2-a]benzimidazole (3a-i)}

To a solution of chalcone (1a-i) in n-butanol, 2-amino benzimidazole (2) was added and reflux the reaction mixture at reflux temperature for 8-10 hrs. After cool down the reaction mixture at room temperature, filtered the solid crude product. Wash the crude product with diethyl ether and dried in vacuo to obtained analytical pure grade compounds 3a-i. Physical Constants newly synthesized of pyrimidine derivatives $3 \mathrm{a}-3 \mathrm{i}$ are recorded in Table 1 .<smiles>[R]c1cccc(C(=O)/C=C/c2ccco2)c1</smiles>

1a-i<smiles>Nc1nc2ccccc2[nH]1</smiles>

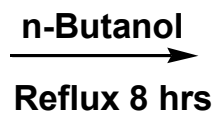<smiles>C1=C(c2ccco2)Nc2nc3ccccc3n2C1c1ccco1</smiles>

3a-i 


\section{3. Spectral characterization}

4-(furan-2-yl)-2-(4-chlorophenyl)-1,4-dihydro pyrimido[1,2-a]benzimidazole (3b) IR (KBr) $v\left(\mathrm{~cm}^{-1}\right): 3047,2945,2874,2818,1620,1560,1508,1460,1276,1172,1008,910,796$, 742, 662, 601, $501 \mathrm{~cm}^{-1},{ }_{1}^{1} \mathrm{H}$ NMR (DMSO-D $) \delta 9.76(\mathrm{~s}, 1 \mathrm{H}), 7.73(\mathrm{dd}, 1 \mathrm{H}), 7.58(\mathrm{dd}, 1 \mathrm{H})$, $7.50-7.39(\mathrm{~m}, 5 \mathrm{H}), 7.24(\mathrm{td}, 1 \mathrm{H}), 7.15(\mathrm{td}, 1 \mathrm{H}), 6.38(\mathrm{t}, 1 \mathrm{H}), 6.37-6.29(\mathrm{~m}, 2 \mathrm{H}), 5.20(\mathrm{~d}$, $1 \mathrm{H}) ; \mathrm{M}^{+}=348$.

Table 1. Physical Constant table of pyrimido[1,2-a]benzimidazole derivatives (3a-3i).

\begin{tabular}{|c|c|c|c|c|c|c|}
\hline No & Comp. & $\mathbf{R}$ & $\begin{array}{c}\text { Molecular } \\
\text { Formula }\end{array}$ & $\begin{array}{c}\text { Molecular } \\
\text { Weight }\end{array}$ & $\begin{array}{c}\text { Yield } \\
(\%)\end{array}$ & $\begin{array}{c}\text { M.P. } \\
\text { (\%) }\end{array}$ \\
\hline 1 & $3 \mathrm{a}$ & $\mathrm{H}$ & $\mathrm{C}_{20} \mathrm{H}_{15} \mathrm{~N}_{3} \mathrm{O}$ & 313 & 60 & 192 \\
\hline 2 & $3 \mathrm{~b}$ & $4-\mathrm{Cl}$ & $\mathrm{C}_{20} \mathrm{H}_{14} \mathrm{ClN}_{3} \mathrm{O}$ & 348 & 75 & 213 \\
\hline 3 & $3 \mathrm{c}$ & $4-\mathrm{Br}$ & $\mathrm{C}_{20} \mathrm{H}_{14} \mathrm{BrN}_{3} \mathrm{O}$ & 392 & 70 & 225 \\
\hline 4 & $3 \mathrm{~d}$ & $4-\mathrm{OCH}_{3}$ & $\mathrm{C}_{21} \mathrm{H}_{17} \mathrm{~N}_{3} \mathrm{O}_{2}$ & 343 & 78 & 185 \\
\hline 5 & $3 \mathrm{e}$ & $4-\mathrm{CH}_{3}$ & $\mathrm{C}_{21} \mathrm{H}_{17} \mathrm{~N}_{3} \mathrm{O}$ & 327 & 82 & 178 \\
\hline 6 & $3 \mathrm{f}$ & $4-\mathrm{NO}_{2}$ & $\mathrm{C}_{20} \mathrm{H}_{14} \mathrm{~N}_{4} \mathrm{O}_{3}$ & 358 & 55 & 230 \\
\hline 7 & $3 \mathrm{~g}$ & $2-\mathrm{Cl}$ & $\mathrm{C}_{20} \mathrm{H}_{14} \mathrm{ClN}_{3} \mathrm{O}$ & 348 & 70 & 240 \\
\hline 8 & $3 \mathrm{~h}$ & $3-\mathrm{Br}$ & $\mathrm{C}_{20} \mathrm{H}_{14} \mathrm{BrN}_{3} \mathrm{O}$ & 392 & 77 & 198 \\
\hline 9 & $3 \mathrm{i}$ & $3,4-(\mathrm{OCH})_{2}$ & $\mathrm{C}_{22} \mathrm{H}_{19} \mathrm{~N}_{3} \mathrm{O}_{3}$ & 373 & 60 & 227 \\
\hline
\end{tabular}

\section{ANTI MICROBIAL ACTIVITY}

The antimicrobial activity was assay by using the disc diffusion method. Newly synthesized compounds were screened in vitro for their antimicrobial activity against four bacterial strains, i.e. two gram +ve baceteria Staphylococcus aureus and Staphylococcus epidermidis and two gram -ve bacteria Escherichia coli and Pseudomonas aeruginosa and fungi strain Aspergillus niger. Standard drug Cephalexin and Greseofulvin were used for the comparison purpose. The obtained results for compounds 3a-3i are recorded Table 2.

Table 2. Antimicrobial activity of pyrimido[1,2-a]benzimidazole derivatives (3a-3i).

\begin{tabular}{|c|c|c|c|c|c|}
\hline \multirow{2}{*}{$\begin{array}{c}\text { Compound } \\
\text { No. } \\
\text { (substitution) }\end{array}$} & \multicolumn{4}{|c|}{ Antibacterial activity (\%) } & $\begin{array}{c}\text { Antifungal } \\
\text { activity (\%) }\end{array}$ \\
\cline { 2 - 6 } & S. aureus & S. epidermidis & E. coli & P. aeruginosa & A. niger \\
\hline $3 \mathrm{a}(\mathrm{H})$ & 80 & 33 & 50 & 67 & 50 \\
\hline $3 \mathrm{~b}(4-\mathrm{Cl})$ & 70 & 71 & 55 & 67 & 63 \\
\hline
\end{tabular}




\begin{tabular}{|c|c|c|c|c|c|}
\hline $3 \mathrm{c}(4-\mathrm{Br})$ & 60 & 54 & 86 & 48 & 67 \\
\hline $3 \mathrm{~d}\left(4-\mathrm{OCH}_{3}\right)$ & 68 & 69 & 68 & 81 & 83 \\
\hline $3 \mathrm{e}\left(4-\mathrm{CH}_{3}\right)$ & 85 & 83 & 45 & 89 & 46 \\
\hline $3 \mathrm{f}\left(4-\mathrm{NO}_{2}\right)$ & 75 & 54 & 85 & 86 & 38 \\
\hline $3 \mathrm{~g}(2-\mathrm{Cl})$ & 90 & 38 & 77 & 80 & 54 \\
\hline $3 \mathrm{~h}(3-\mathrm{Br})$ & 50 & 79 & 55 & 52 & 75 \\
\hline $3 \mathrm{i}\left(3,4-(\mathrm{OCH})_{2}\right.$ & 45 & 42 & 64 & 67 & 71 \\
\hline Amoxicillin & 100 & 100 & 100 & 100 & - \\
\hline Greseofulvin & - & - & - & - & 100 \\
\hline
\end{tabular}

\section{CONCLUSION}

In present report, we submitted very efficient method for the synthesis of some new pyrimido[1,2-a]benzimidazole derivatives without use of any catalyst. All synthesized compounds were obtained in good to moderate yield. The synthesized compounds were characterized by ${ }^{1} \mathrm{H}$ NMR, Mass and IR spectroscopy and the obtained results are showing good agreement with the synthesized structures.

From the results of antimicrobial data, compounds $3 \mathrm{e}$ and $3 \mathrm{~g}$ were shown good activity against bacterial pathogens while compounds $3 \mathrm{~d}, 3 \mathrm{~h}$ and $3 \mathrm{i}$ were found good active against fungi pathogens as compare to the standard drugs.

\section{Acknowledgement}

The author are thankful for facilities and grants given under UGC - SAP (DRS - Department Research Support)540/6/DRS/2004 (SAP-1) Dt. 26-03-2004 and Department of Science and Technology, New Delhi, for fund for improvement of Science and Technology (FIST-sanction no. SR/FIST/CSI/072/2003 date. 24-12-2003). Nirav Shah is also thankful to University Grant Commission for providing UGC-BSR Meritorious Fellowship.

\section{References}

[1] Q. McKellar, E. Scott, J. Vet. Pharmacol. Ther. 13 (1990) 223.

[2] A. Spasov, I. Yozhitsa, L. Bugaeva, V. Anisimova, Pharm. Chem. J. 33 (1999) 232.

[3] J. Rossignol, H. Maisonneuve, Ann. Trop. Med. Parasitol. 78 (1984) 135

[4] A. Patil, S. Ganguly, S. Surana, Rasayan J. Chem. 1 (2008) 447.

[5] A. Dubey, P. Sanyal, Online Vet. J. 5 (2010) 63.

[6] M. Boiani, M. Gonzalez, Mini Rev. Med. Chem. 5 (2005) 409.

[7] B. Narasimhan, D. Sharma, P. Kumar, Med. Chem. Res. 21 (2012) 269. 
[8] Y. Fellahi, P. Dubois, V. Agafonov, F. Moussa, J. E. Ombetta-Goka, J. Guenzet, Y. Frangin, Bull. Soc. Chim. Fr. 133 (1996) 869.

[9] J. Kempson, et. al., Bioorg. Med. Chem. Lett. 15 (2005) 1829.

[10] V. Chebanov, S. Desenko, Curr Org Chem 10 (2006) 297.

[11] S. Desenko, Chem Heterocycl Comp 31 (1995) 125.

[12] P. Zalavadiya, S. Tala, J. Akbari, H. Joshi, Archiv der Pharmazie 342 (2009) 469.

[13] R. Khunt, J. Akbari, A. Manvar, S. Tala, M. Dhaduk, H. Joshi, A. Shah, Arkivoc 11 (2008) 277.

[14] P. Zalavadiya, R. Ghetiya, B. Dodiya, P.Vekariya, H. Joshi, Journal of Heterocyclic Chemistry 50 (2013) 973.

[15] S. Rokad, S. Tala, J. Akbari, M. Dhaduk, H. Joshi, Journal of the Indian Chemical Society 86 (2009) 186.

[16] S. Tala, P. Vekariya, R. Ghetiya, B. Dodiya, H. Joshi, Indian Journal of Chemistry 52 (2013) 807.

[17] G. Thirunarayanan, M. Suresh, International Letters of Chemistry, Physics and Astronomy 4 (1) (2014) 1-11.

[18] K. G Sekar, G. Thirunarayanan, International Letters of Chemistry, Physics and Astronomy 8(3) (2013)249-258.

[19] G. Thirunarayanan, International Letters of Chemistry, Physics and Astronomy 5 (2014) 89-98.

[20] K. G. Sekar, G. Thirunarayanan, International Letters of Chemistry, Physics and Astronomy 8(2) (2013) 160-174.

[21] G. Thirunarayanan, International Letters of Chemistry, Physics and Astronomy 4 (2014) 109-116. 